\title{
Rationally designed nanocarriers for intranasal therapy of allergic rhinitis: influence of carrier type on in vivo nasal deposition
}

This article was published in the following Dove Press journal:

International Journal of Nanomedicine

25 May 2016

Number of times this article has been viewed

\author{
Marwa Ahmed Sallam \\ Hala Mahmoud Helal \\ Sana Mohamed Mortada \\ Department of Industrial Pharmacy, \\ Faculty of Pharmacy, Alexandria \\ University, Alexandria, Egypt
}

\begin{abstract}
The aim of this study is to develop a locally acting nasal delivery system of triamcinolone acetonide (TA) for the maintenance therapy of allergic rhinitis. The effect of encapsulating TA in different nanocarriers on its mucosal permeation and retention as well as in vivo nasal deposition has been studied. A comparative study was established between polymeric oil core nanocapsules (NCs), lipid nanocarriers such as nanoemulsion (NE), and nanostructured lipid carriers (NLCs). The elaborated nanocarriers were compared with TA suspension and the commercially available suspension "Nasacort ${ }^{\mathbb{R} \text { ". }}$. The study revealed that NC provided the highest mucosal retention, as $46.14 \% \pm 0.048 \%$ of the TA initial dose was retained after 24 hours, while showing the least permeation through the nasal mucosa. On the other hand, for TA suspension and Nasacort ${ }^{\circledR}$, the mucosal retention did not exceed $23.5 \% \pm 0.047 \%$ of the initial dose after 24 hours. For NE and NLC, values of mucosal retention were $19.4 \% \pm 0.041 \%$ and $10.97 \% \pm 0.13 \%$, respectively. $\mathrm{NC}$ also showed lower mucosal irritation and superior stability compared with NE. The in vivo nasal deposition study demonstrated that NC maintained drug in its site of action (nasal cavity mucosa) for the longest period of time. The elaborated polymeric oil core NCs are efficient carriers for the administration of nasally acting TA as it produced the least permeation results, thus decreasing systemic absorption of TA. Although NCs have been administered via various routes, this is the first study to implement the polymeric oil core NC as an efficient carrier for localized nasal drug delivery.
\end{abstract}

Keywords: allergic rhinitis, nanocapsules, triamcinolone acetonide, nasal mucosa, nasal deposition

\section{Introduction}

Allergic rhinitis (AR) is one of the most common health problems having a negative impact on the quality of life. It is characterized by nasal symptoms including rhinorrhea, itching, and sneezing. In addition, nasal congestion, which is a main symptom of AR or sinusitis, interferes with the ability to speak and hear or can even cause life-threatening sleep apnea. ${ }^{1,2}$ AR involves nasal inflammation that results from IgE-mediated immune responses to specific allergens. Corticosteroids are one of the main treatment options that prevent or suppress inflammation and immune responses. They are used in the treatment of allergic and nonallergic mucosal inflammation and also rhinitis, sinusitis, and asthma-combined nasal rhinitis. However, their therapeutic outcome is often unacceptable because of their poor distribution in the nose and sinuses.

The prolonged residual time of steroids in the nasal cavity is also a crucial factor in treating rhinitis and nasal congestion. Nanotechnology-based approaches appeared 
to increase the efficiency of locally acting nasal steroids by increasing the residence time of the therapeutic agent in the nasal cavity. ${ }^{3}$

Thus, different techniques were applied in order to ensure in situ action of nasal drugs. Environment-sensitive hydrogels and thermosensitive gels have been developed to ensure the purpose of action. ${ }^{4,5}$

Triamcinolone acetonide (TA) is a synthetic corticosteroid administered as a nasal spray $\left(\right.$ Nasacort $^{\circledR} \mathrm{AQ}$, Sanofi, Bridgewater, NJ, USA) for relieving the symptoms of rhinitis. ${ }^{6}$ There are some disadvantages with the commercially available spray, including the common side effects such as nasal irritation and epistaxis, frequent administration, and bitter taste (through connection between nose and mouth). This is in addition to the decrease in the drug residence in nostril by defense mechanisms as mucociliary clearance. With a beat frequency of $\sim 1,000$ strokes/min, the cilia transport the mucus to the nasopharynx at a speed of $5 \mathrm{~mm} / \mathrm{min}$. Accordingly, formulations on the mucosa are rapidly eliminated from the nasal cavity, with a half-life of $\sim 15$ minutes. The rapid clearance of formulations is the biggest challenge for nasal drug delivery. In addition, $\sim 50 \%$ of the TA is absorbed into the blood. The usage of this drug is controversial in some patients such as pregnant or breast-feeding women. ${ }^{7}$ These findings highlight the need for new drug delivery strategies. In this case, it is required to deliver the drug directly to the site of action for minimizing the systemic side effects.

This work aims to provide a nonirritant, locally acting nasal drug delivery system intended for the treatment of AR and prophylaxis of asthma. Different TA-loaded nanoparticulate systems were prepared, including oil core nanocapsules (NCs), nanoemulsion (NE), and nanostructured lipid carriers (NLCs), to select the most appropriate nanocarrier system for the preparation of the locally acting TA with minimal systemic absorption. The elaborated nanocarriers were characterized regarding colloidal properties, their mucosal retention, sustaining the drug release, mucoadhesiveness, and in vivo nasal deposition, in comparison with the drug suspension and commercially available Nasacort ${ }^{\circledR}$. The ciliotoxicity of prepared formulations was evaluated by histopathological examination of the treated nasal mucosa.

\section{Materials and methods}

\section{Materials}

TA was obtained from TCI Europe N.V. (Zwijndrecht, Belgium). Poloxamer ${ }^{\circledR} 188$ was purchased from SigmaAldrich (St Louis, MO, USA). Capryol $^{\circledR}$ and Gelucire ${ }^{\circledR}$
50/13 were gift samples from Gattefossé (Nanterre, France). Miglyol ${ }^{\circledR}$ was from Sasol (Hamburg, Germany). Eudragit ${ }^{\circledR}$ RS100 was obtained from Evonik (Essen, Germany). Lipoid ${ }^{\circledR}$ S75 was a gift sample from Lipoid ${ }^{\circledR}$, Ludwigshafen, Germany. All other materials were of analytical grade. Mucin from porcine stomach (Type III, bound sialic acid $0.5 \%-1.5 \%$, partially purified powder) was purchased from Sigma-Aldrich.

\section{Methods}

High-performance liquid chromatography assay of TA A reversed-phase C18 analytical column (220×4.4 mm ID, $5 \mu \mathrm{m}$ particle size) and a mobile phase of water:acetonitrile (50:50, v/v) were used for high-performance liquid chromatography (HPLC) analysis of TA. All solvents were degassed for 10 minutes before use. The flow rate was $1 \mathrm{~mL} / \mathrm{min}$. The injection volume was $50 \mu \mathrm{L}$, and the effluent was monitored at $240 \mathrm{~nm}$ by UV detector. The retention time was $\sim 5.62 \pm 0.46$ minutes. Elution was carried out isocratically at ambient temperature. The calibration curve was linear over the range of $2.5-20 \mu \mathrm{g} / \mathrm{mL}$. Under these conditions, the drug concentration was determined using the equation $Y=10,97,930 X+1,09,114$, where $Y$ is the peak area and $X$ is the drug concentration in $\mu \mathrm{g} / \mathrm{mL}$. The method was selective with no interference from other formulation components. ${ }^{8}$ The limit of detection was $0.2 \mu \mathrm{g} / \mathrm{mL}$, and the limit of quantitation was $0.7 \mu \mathrm{g} / \mathrm{mL}$.

\section{Solubility study of TA}

The solubility of TA was determined in Miglyol $^{\circledR}$, Capryol $^{\circledR}$, Labrafac $^{\circledR}$, distilled water, $0.1 \mathrm{M} \mathrm{HCl}$, and phosphate-buffered saline (PBS) pH 7.4. A known excess amount of TA was added to a stoppered glass vial containing $1 \mathrm{~mL}$ of each of the tested vehicles. The samples were sonicated (Julabo sonicator, USR, Seelbach, Germany) for 10 minutes at $25^{\circ} \mathrm{C}$ and left in a thermostatically controlled shaking water bath for 24 hours at $25^{\circ} \mathrm{C} \pm 0.5^{\circ} \mathrm{C}$. This was followed by equilibrium for 2 hours. Mixtures were centrifuged at 4,000 rpm for 10 minutes (80-2 electric centrifuge, Jiangsu Jintan Medical Instrument Co., Ltd., Jiangsu, People's Republic of China), followed by filtration of the supernatant through a Millipore membrane filter (0.45 $\mu \mathrm{m}$ HAWP 074700, EMD Millipore, Billerica, MA, USA). Samples from the filtrate were suitably diluted with methanol and quantified spectrophotometrically (T80 UV/VIS spectrophotometer, PG Instruments Ltd., Lutterworth, UK) for the dissolved TA at $238 \mathrm{~nm}$ against a similar dilution of the plain vehicle in methanol as a blank solution. The experiment was performed in triplicate. 
The results were expressed as mean value $(\mathrm{mg} / \mathrm{mL}) \pm$ standard deviation (SD).

\section{Preparation of TA-loaded nanocarriers}

Different nanocarriers of TA were prepared including oil core NC, NE, and NLC. NCs were prepared by the nanoprecipitation method. ${ }^{9}$ An organic phase consisting of $0.1 \mathrm{~g}$ of Eudragit RS100, $1 \mathrm{~mL}$ of Capryol ${ }^{\circledR}$, and $6 \mathrm{mg}$ of TA dissolved in a $20 \mathrm{~mL}$ mixture of acetone:ethanol (3:1) was injected to an aqueous phase of $40 \mathrm{~mL}$ of distilled water containing $1 \mathrm{~g}$ of Poloxamer 188 as a stabilizer under magnetic stirring at $300 \mathrm{rpm}$ for 15 minutes. The observation of a bluish white emulsion indicated the formation of NC. The formed emulsion was left overnight under magnetic stirring to ensure complete elimination of the organic solvents.

For the preparation of TA-loaded NE, the procedure was the same as in the preparation of $\mathrm{NC}$, but without the addition of Eudragit RS100 in the organic phase.

TA-loaded NLCs were prepared by the homogenizationsonication technique described by Mendes et al ${ }^{10}$ with a slight modification. In brief, the organic phase was composed of $400 \mathrm{mg}$ Gelucire $^{\circledR} 50 / 13,200 \mu \mathrm{L} \mathrm{Capryol}^{\circledR}$, $1.2 \mathrm{mg} \mathrm{TA}$, and $400 \mathrm{mg}$ Lipoid $^{\circledR} \mathrm{S} 75$ dissolved in $5 \mathrm{~mL}$ ethanol at a temperature of $5^{\circ} \mathrm{C}$ above the melting point of solid lipid. An aqueous phase of $20 \mathrm{~mL}$ distilled water containing $400 \mathrm{mg}$ Poloxamer 188 was heated to the same temperature as the organic phase and added dropwise to the organic phase under high-speed homogenization (ULTRATURRAX T25, IKA Labortechnik, Straufen, Germany) at $10,000 \mathrm{rpm}$ for 15 minutes. This was followed by sonication (Julabo sonicator, USR, Seelbach, Germany) at the same temperature for 45 minutes. The formed oil-in-water emulsion was stirred at room temperature to allow cooling and for the formation of NLC.

\section{Characterization of TA-loaded nanocarriers \\ Particle size, polydispersity index, and zeta potential}

The mean particle size ( $Z$-average size), polydispersity index (PDI), and zeta potential (ZP) were measured using a dynamic light scattering (DLS) technique with a NanoZS/ ZEN3600 Zetasizer (Malvern Instruments, Malvern, UK). This system is equipped with a $4-\mathrm{mW}$ helium/neon laser at $633 \mathrm{~nm}$ wavelength and measures the particle size with the noninvasive backscattering technology at a detection angle of $173^{\circ}$. Before measurement, NC, NE, and NLC dispersions were diluted with purified water (1:20) to avoid the multidispersion of the light caused by a high concentration of particles. All the measurements were performed in triplicate.

\section{Encapsulation efficiency}

The encapsulation efficiency of the three nanocarriers was determined by an equilibrium dialysis method using dialysis membranes (Mwt cutoff 12,000-14,000 Spectra-Por, Spectrum Labs., Irving, TX, USA). ${ }^{11}$ Here, $5 \mathrm{~mL}$ of each of the investigated formulations containing $1.75 \mathrm{mg}$ TA was dialyzed against PBS PH 7.4 for 2 hours at $8^{\circ} \mathrm{C}$. The encapsulation efficiency was calculated as follows:

$$
\% \mathrm{EE}=\frac{\begin{array}{l}
\text { Initial amount of TA } \\
\text { in the formulation }
\end{array}-\begin{array}{c}
\text { Amount of TA } \\
\text { dialyzed in } 2 \text { hour }
\end{array}}{\text { Initial amount of TA in the formulation }} \times 100
$$

\section{Morphological analysis by transmission electron microscope}

The morphology of the NC, NE, and NLC was observed by transmission electron microscopy (TEM; Jeol, JEM-100 CX, Joel, Tokyo, Japan). The sample was diluted with water (1:3), and a drop was placed on a copper grid. The excess was drawn off with a filter paper. Samples were subsequently stained with uranyl acetate solution for 30 seconds and then naturally dried.

\section{In vitro drug release}

Approximately $10 \mathrm{~mL}$ of each of the investigated formulations was placed inside a dialysis bag (Mwt cutoff $12-14 \mathrm{kDa}$ ), which was placed in $125 \mathrm{~mL}$ PBS $(\mathrm{pH} 7.4)$ at $37^{\circ} \mathrm{C} \pm 0.5^{\circ} \mathrm{C}$ and allowed to rotate in a rotating paddle dissolution apparatus (Pharma test, PTWS 3, Pharma Test Apparatebau AG, Hainburg, Germany) at $100 \mathrm{rpm} .{ }^{12}$ In comparison, the drug suspension was dialyzed against the same dissolution medium. Aliquots of $5 \mathrm{~mL}$ were removed at predetermined time intervals $(0.5,1,2,4,6,8$, and 24 hours $)$ and replaced by fresh PBS. The samples were filtered through the $0.45-\mu \mathrm{m}$ Millipore filter and analyzed quantitatively for TA (T80 UV/ VIS spectrophotometer, PG Instruments Ltd.).

\section{Preparation and characterization of a powder form of TA-NC}

The NC dispersion containing $6 \mathrm{mg}$ TA was lyophilized (Cryodos-50 Freeze-drier, Telstar Cryodos, Terrassa, Spain) in the presence of $5 \% \mathrm{w} / \mathrm{v}$ mannitol as cryoprotectant to obtain the free-flowing TA-loaded NC powder. 
The recovery yield was calculated according to the following equation: ${ }^{13}$

$$
\text { Yield }=\frac{\text { Mass of nanoparticles recovered }}{\text { Mass of polymer }+ \text { TA }+ \text { Excipients }} \times 100
$$

To determine the TA content, an accurately weighed amount of the freeze-dried TA-loaded NC containing theoretically $1 \mathrm{mg}$ of TA was sonicated for 30 minutes in $5 \mathrm{~mL}$ methanol to ensure complete dissolution of the encapsulated TA. This solution was then filtered using the Millipore membrane filter (0.45 $\mu \mathrm{m}$ HAWP 074700; EMD Millipore) and assayed spectrophotometrically at $238 \mathrm{~nm}$. The experiment was carried out in duplicate, and the percentage drug loading (\%DL) was calculated using the following equation:

$$
\% \text { Drug loading }=\frac{\text { Mass of } \mathrm{TA} \text { in } \mathrm{NC}}{\text { Mass of } \mathrm{NC} \text { recovered }} \times 100
$$

\section{Differential scanning calorimetry}

The differential scanning calorimetry (DSC) thermograms of pure TA, polymer, and TA-loaded NC as well as their respective physical mixture were recorded using the DSC6 differential scanning calorimeter (PerkinElmer Inc., Waltham, MA, USA). Approximately $6 \mathrm{mg}$ of each sample was placed in sealed aluminum pans and heated at $10^{\circ} \mathrm{C} / \mathrm{min}$ under a nitrogen atmosphere in the $30^{\circ} \mathrm{C}-400^{\circ} \mathrm{C}$ range. An empty aluminum pan was used as a reference. The equipment was periodically calibrated with indium. ${ }^{8}$

\section{Fourier transform infrared spectra}

The Fourier transform infrared (FTIR) spectra of polymer, TA, Poloxamer ${ }^{\circledR} 188$, their respective physical mixture, and TA-loaded NC were recorded. Samples were finely grounded with IR grade $\mathrm{KBr}$ and then pressed into pellet, and IR spectra were taken in transmission over the range of $4,000-500 \mathrm{~cm}^{-1}$ at ambient temperature.

\section{Ex vivo bovine nasal mucosal retention}

In brief, bovine nasal mucosae were obtained from freshly slaughtered cattle. The skin covering the nose was removed, and the frontal part of the nasal conchae (conchae nasales dorsales) was cut with a sharp scalpel, starting from the incisura nasoincisiva. The mucosal tissue was then carefully stripped from the lateral cartilage using a pair of tweezers. ${ }^{14}$

The freshly excised bovine nasal mucosa of thickness $0.12 \mathrm{~cm}$, excluding the septum part, was collected and used for the permeation study. The permeation of TA from the elaborated NC, NE, and NLC as well as TA suspension through the bovine nasal mucosa was carried out using the vertical Franz diffusion cell. The mucosal specimen was mounted on the diffusion cell of effective surface area $3.14 \mathrm{~cm}^{2}$, with mucosal surface facing the donor chamber and serosal side facing the receptor chamber. The latter was then filled with 8 $\mathrm{mL}$ PBS ( $\mathrm{pH}$ 7.4), with great care taken to avoid trapping air beneath the mucosa. The cell was submerged in a thermostatically controlled shaker water bath (SBS40Stuart, Staffordshire, UK) operated at 50 strokes $/ \mathrm{min}$ at $37^{\circ} \mathrm{C}$. After an equilibration time with PBS for 30 minutes, the permeability experiments were started by replacing the media in a receiver chamber with $8 \mathrm{~mL}$ fresh $\mathrm{PBS} \mathrm{pH} 7.4\left(37^{\circ} \mathrm{C}\right)$ and that of the donor chamber with $100 \mu \mathrm{L}$ (equivalent to $0.032 \mathrm{mg}$ of TA) of each of the tested formulations. ${ }^{15}$ At predetermined time intervals $(2,4,6$, 8 , and 24 hours), the entire receptor medium was withdrawn from the receiver compartment and replenished with an equal volume of fresh $\mathrm{PBS}$ at $37^{\circ} \mathrm{C}$. Samples from the receptor media were filtered through the $0.45-\mu \mathrm{m}$ Millipore filter analyzed for TA using the HPLC (PerkinElmer Inc.). The cumulative amount of TA permeated per unit area of nasal mucosa was plotted against time. After 24 hours, the mucosal surface was washed with PBS to remove any remaining formulation. To determine the amount of TA entrapped in the mucosa, the mucosa was sonicated with a known amount of ethanol, which was then analyzed for TA content using the previously reported HPLC method.

\section{Histopathological assessment of local toxicity on nasal mucosa}

Histopathological studies were carried out using the bovine nasal mucosa with uniform thickness mounted on Franz diffusion cells. The studies were performed on mucosa treated with TA suspension and TA-loaded nanocarrier formulations as well as mucosa treated with $0.5 \mathrm{~mL}$ of PBS pH 7.4 and isopropyl alcohol. After 2 hours, the mucosa was rinsed with PBS pH 7.4 and was fixed in $10 \%$ formalin, routinely processed, and embedded in paraffin. Paraffin sections were cut and placed on glass slides and stained with hematoxylin and eosin. The bovine nasal mucosae treated with PBS pH 7.4 and isopropyl alcohol were taken as negative and positive controls, respectively. Sections were analyzed by a pathologist blinded to the experimental conditions. ${ }^{16}$

\section{Assessment of nanocarriers-mucin interaction}

The mucoadhesive tendency of elaborated nanocarriers, drug suspension, and marketed Nasacort ${ }^{\circledR}$ formulation was evaluated by the turbidimetric assessment of the mucin/ particle interactions. In brief, the mucin powder was dispersed 
in water $(0.1 \% \mathrm{w} / \mathrm{v})$ and stirred overnight. The dispersion was filtered twice through a $0.45-\mu \mathrm{m}$ Millipore filter to collect the mucin-containing supernatant. Equal volumes of mucin dispersion $(0.1 \% \mathrm{w} / \mathrm{v})$ and the nanocarrier dispersion were mixed together by vortexing for 1 minute. The turbidity of the mixtures was measured at zero time and after incubation for 2 hours at $37^{\circ} \mathrm{C}$. The absorbance was recorded at $650 \mathrm{~nm}$ using a spectrophotometer (T80 UV/VIS spectrophotometer, PG Instruments Ltd.).

The particle size and ZP of the nanocarrier dispersions in mucin were assessed after dilution with water. Spectrophotometric turbidity determination, particle size, and ZP measurements were performed every 30 minutes for 2 hours to determine the mucin-nanoparticles interaction. ${ }^{17,18}$ The experiments were run in triplicate. The results were expressed as mean \pm SD. For testing the possible interaction between mucin solution and each of TA and Nasacort ${ }^{\circledR}$ dispersion, only the $\mathrm{ZP}$ of the blend at $37^{\circ} \mathrm{C}$ was accessed at different time intervals from incubation and was compared with the ZP of the mucin solution.

In vivo nasal drug deposition testing

The in vivo nasal deposition method adopted by Faraj et al ${ }^{19}$ with slight modification was used.

\section{Animal surgical technique}

Five groups of six rats each weighing 200-230 g were anesthetized by intraperitoneal injection of thiopental solution at a dose of $0.1 \mathrm{~mL} / 100 \mathrm{~g}$ rat body weight. An incision was made in the cervical region, and the trachea was cannulated with a polyethylene tube to maintain respiration. Esophagus was threaded to keep the formulations in the nasal cavity and prevent oral absorption. The nasopalatine was closed with an adhesive agent to prevent drainage of the drug from the nasal cavity to the mouth.

Experiments were performed in accordance with the European Community guidelines for the use of experimental animals and were approved by the Ethics Committee of the Faculty of Pharmacy, Alexandria University.

\section{TA deposition in the nasal cavity}

Approximately $40 \mu \mathrm{L}$ of TA-loaded NC, NE, and NLC, TA suspension, and Nasacort ${ }^{\circledR}$ containing $32 \mu \mathrm{g}$ of TA were introduced in one of the nostrils of each rat with a micropipette. After specified times of deposition (10, 20, 30, 40, 50, and 60 minutes), the residual drug in the nasal cavity was recovered by washing thoroughly with $2 \mathrm{~mL}$ of physiological saline $\mathrm{pH} 7.4$ at a flow rate of $1 \mathrm{~mL} / \mathrm{min}$. The profiles of drug clearance were determined from the amounts of drug in the lavage fluid. Drug concentrations in the lavage fluid were determined by HPLC after filtration with the $0.22-\mu \mathrm{m}$ Millipore filter. ${ }^{20}$ The experiment was performed on six rats for each of the investigated systems, and the results were expressed as mean $\pm \mathrm{SD}$.

\section{Stability of TA-loaded nanocarriers}

Stability studies were carried out for the prepared nanocarriers NC, NE, and NLC over a period of 3 months. The nanocarriers were stored in glass vials sealed with plastic caps and were kept in the fridge at a temperature of $4^{\circ} \mathrm{C}-8^{\circ} \mathrm{C}$. The formulations were monitored for changes in morphology, particle size, ZP, and PDI as well as the chemical stability (drug content).

\section{Results and discussion}

Nasal administration of corticosteroids as a local treatment showed better behavior over oral leukotriene antagonists in ceasing seasonal AR accompanied with asthma, especially in the upper airway. ${ }^{21}$ In this study, we aimed to provide a nanocarrier system for TA capable of achieving appropriate nasal mucosal retention of the drug in an attempt to improve the quality of local treatment and prophylaxis of rhinitis accompanied with asthma. Lipid-core polymeric NC, NE, and NLC were investigated as nanocarriers for TA to select the most appropriate carrier providing nasal retention of the drug within the nasal cavity.

\section{Solubility of TA}

The solubility of TA was found to be $6.5 \pm 0.212,1.36 \pm 0.197$, and $1.37 \pm 0.226 \mathrm{mg} / \mathrm{mL}$ in Capryol $^{\circledR}$, Miglyol $^{\circledR}$ and Labrafac ${ }^{\circledR}$, respectively. Accordingly, Capryol ${ }^{\circledR}$ was the oil selected for the preparation of TA-loaded NC, NE, and NLC.

\section{Characterization TA-loaded nanocarriers}

The mean diameter, PDI, and ZP values of $\mathrm{NC}$, NE, and NLC loaded with TA are given in Table 1. The nanoprecipitation method used in the preparation of NC and the high shear homogenization/ultrasonication method used in the

Table I Particle size, zeta potential (Z), PDI, and EE of TAloaded nanocarriers

\begin{tabular}{lllll}
\hline $\begin{array}{l}\text { Nanometric } \\
\text { system }\end{array}$ & $\begin{array}{l}\text { Size } \\
(\mathbf{d} . \mathbf{n m})\end{array}$ & $\begin{array}{l}\mathbf{Z} \\
(\mathbf{m v})\end{array}$ & PDI & EE\% \pm SD \\
\hline NC & $128.4 \pm 0.503$ & +52.7 & $0.147 \pm 0.006$ & $96.79 \pm 0.36$ \\
NE & $212.1 \pm 2.747$ & -24.7 & $0.130 \pm 0.022$ & $94.15 \pm 0.35$ \\
NLC & $36.25 \pm 0.245$ & -22.2 & $0.266 \pm 0.010$ & $93.05 \pm 0.35$ \\
\hline
\end{tabular}

Abbreviations: $\mathrm{EE}$, encapsulation efficiency; NC, nanocapsule; NE, nanoemulsion; NLC, nanostructured lipid carrier; PDI, polydispersity index; SD, standard deviation; TA, triamcinolone acetonide. 
preparation of NLC resulted in nanocarriers characterized by a mean diameter $\sim 200 \mathrm{~nm}$ and a PDI $<0.2$. This indicates a homogenous particle-size distribution. ${ }^{22}$

The ZP is an important factor affecting the physical stability of colloidal dispersions. Our results showed that the NCs are positively charged, a factor which could be attributed to the quaternary ammonium groups of Eudragit ${ }^{\circledR}$ RS100. ${ }^{23}$ On the other hand, the outer shell of NLC and NE consisted of Gelucire ${ }^{\circledR}$ 50/13 (stearoyl polyoxyl-32 glycerides) and Capryol $^{\circledR} 90$ (propylene glycol monocaprylate type II, >90\%), respectively, where both components carried negative charges accounting for the negative ZP values. In theory, higher values of the ZP, either positive or negative, tend to stabilize the colloid because of the electrostatic repulsion between particles with the same electrical charge. ${ }^{24}$

The encapsulation efficiency of the prepared formulations was $>85 \%$ (Table 1 ). Thus, the oily core of formulations facilitated solubilization of TA with high efficiency. This indicates that nanoencapsulation is an effective technique for improving the solubility of TA in aqueous media.

\section{Morphological analysis by TEM}

Figure 1 shows the TEM micrographs of TA-loaded nanocarriers. NC and NLC showed spherical, well-separated and finely distributed particles. The NE showed a much bigger size with less homogeneity in size distribution. This was in line with the DLS data.

\section{In vitro drug release}

A sustained-release pattern is a crucial parameter in developing nanocarriers for a maintained therapy. The aim of the in vitro drug release study is to compare the TA release profile from different formulations rather than mimicking the drug release in the nasal cavity. All the elaborated nanocarriers demonstrated a prolonged release of TA. On the contrary, 97\% TA was released within the first 4 hours from TA suspension in PBS (Figure 2).

\section{Differential scanning calorimetry}

To confirm the physical state of TA in the freeze-dried NC, DSC analysis was carried out for TA, Eudragit RS100, Poloxamer F188, their physical mixture, and freeze-dried TA-NC (Figure 3).

TA thermogram revealed an endothermic peak, which corresponds to a melting point of $\sim 300^{\circ} \mathrm{C}$, and is indicative of a crystalline anhydrous state for TA. In the physical mixture, all characteristic peaks of all components were detectable at their original positions. The peak of TA disappeared from the thermogram of TA-NC, suggesting that TA was totally encapsulated in NC in an amorphous or solid solution state.

\section{Fourier transform infrared spectra}

FTIR spectra in Figure 4 were recorded to assess the compatibility of the drug and excipients. Eudragit RS100 showed an ester $\mathrm{C}=\mathrm{O}$ stretching peak approximately $1,735.75 \mathrm{~cm}^{-1}$. The empirical formula of TA is $\mathrm{C}_{24} \mathrm{H}_{31} \mathrm{FO}_{6}$, structured as 9-fluoro-11 $\beta, 16 \alpha, 17,21$-tetrahydroxypregna-1,4-diene-3,20dione16,17-cyclic acetal with acetone. In the FTIR spectrum, the characteristic bands observed from the data of TA included the $\mathrm{OH}$ group in the range of 3,650-3,200 $\mathrm{cm}^{-1}, \mathrm{C}-\mathrm{H}$ stretching of sp3 and sp2 carbons in the range of 3,000-2,900 $\mathrm{cm}^{-1}$, $\mathrm{C}=\mathrm{O}$ in $1,775-1,650 \mathrm{~cm}^{-1}, \mathrm{C}=\mathrm{C}$ in $1,690-1,635 \mathrm{~cm}^{-1}, \mathrm{C}-\mathrm{O}-\mathrm{C}$
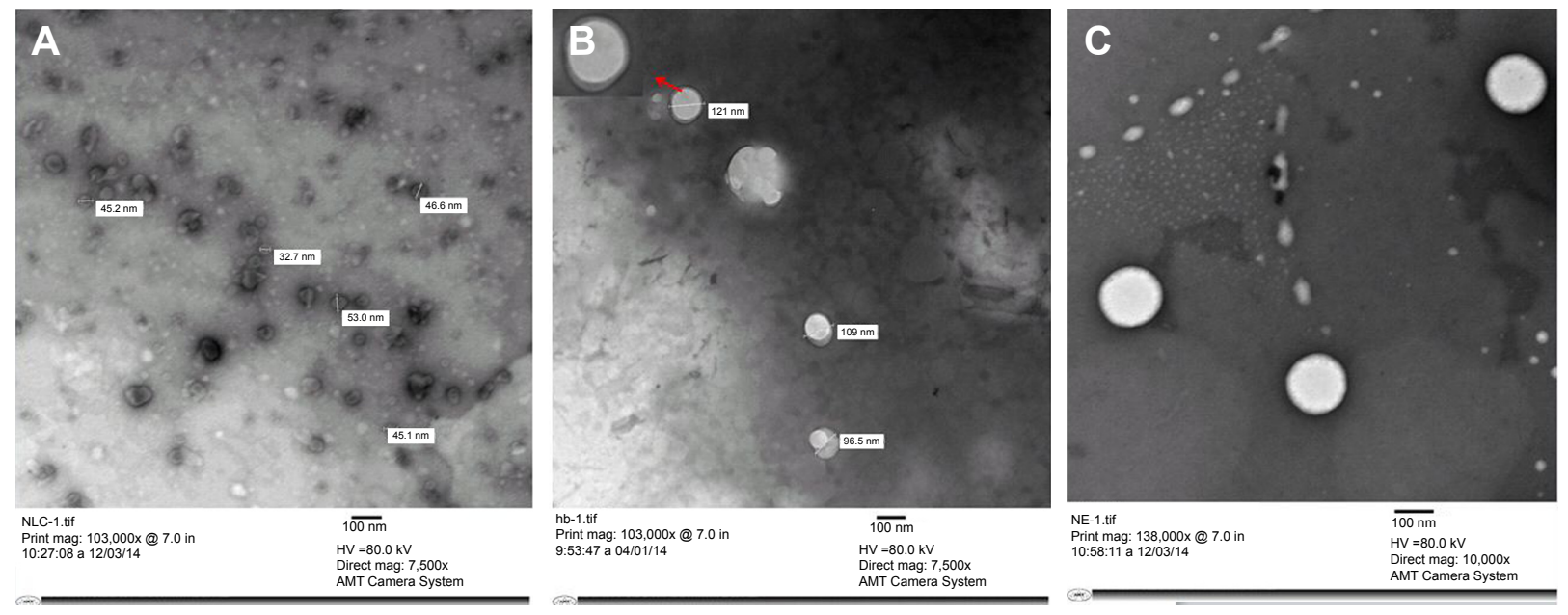

Figure I Transmission electron micrographs of NLC (A), NC (B), and NE (C).

Note: Red arrow shows magnified view of nanocapsule showing core-shell structure.

Abbreviations: NC, nanocapsule; NE, nanoemulsion; NLC, nanostructured lipid carrier. 


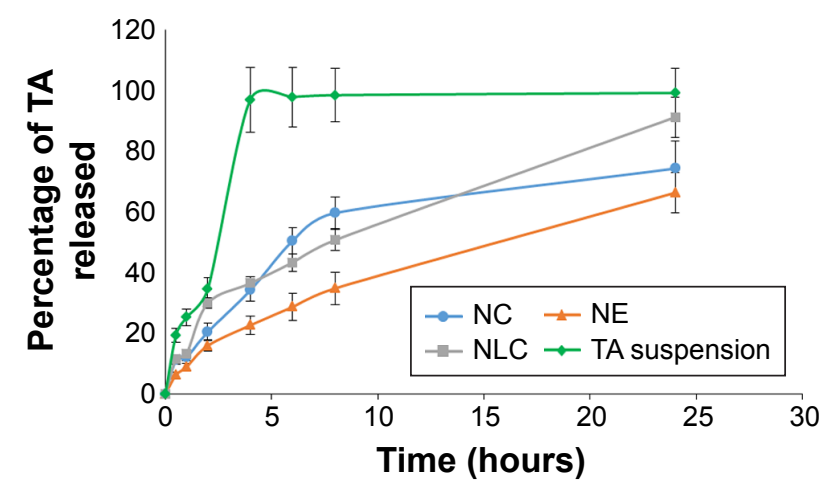

Figure 2 In vitro release of TA from NC, NE, NLC, and TA suspension. Abbreviations: NC, nanocapsule; NE, nanoemulsion; NLC, nanostructured lipid carrier; TA, triamcinolone acetonide.

in $1,310-1,000 \mathrm{~cm}^{-1}$, and a strong peak at $1,058 \mathrm{~cm}^{-1}$ because of stretching vibration of $\mathrm{C}-\mathrm{F} .{ }^{25}$ The IR spectrum of Poloxamer F188 is characterized by the principal absorption peaks at $2,883.38 \mathrm{~cm}^{-1}$ (C-H stretch aliphatic), $1,348.15 \mathrm{~cm}^{-1}$ (in-plane $\mathrm{O}-\mathrm{H}$ bend), and $1,107.06 \mathrm{~cm}^{-1}$ (C-O stretch).

All the characteristic peaks of TA are present in their original positions in the physical mixture as well as in the TA-loaded NC. This showed that the molecular structure of TA remained completely intact, indicating the absence of chemical interaction between TA and any of the ingredients used in the $\mathrm{NC}$ formulation.

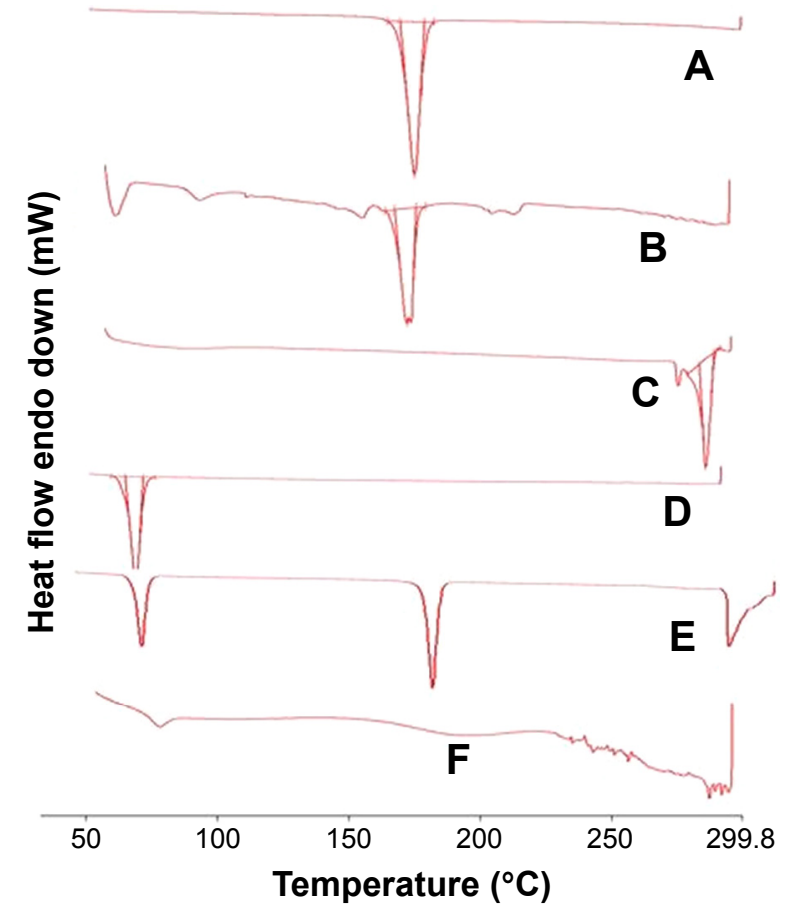

Figure 3 DSC thermograms of mannitol (A), TA NC (B), TA (C), Pluronic FI 27 (D), physical mixture (E), and Eudragit RSI00 (F).

Abbreviations: DSC, differential scanning calorimetry; NC, nanocapsule; TA, triamcinolone acetonide.

\section{Ex vivo bovine nasal mucosal permeation and retention}

Figure 5 illustrates the TA permeation from NC, NE, and NLC, TA suspension, and Nasacort ${ }^{\circledR}$ through bovine nasal mucosa as functions of time. The percentage of TA permeated within 24 hours was 58\% from drug suspension, 40\% from Nasacort $^{\circledR}$, and only $35 \%, 35 \%$, and $37 \%$ for $\mathrm{NC}$, NE, and NLC, respectively. This indicates considerable retardation of permeation of TA by nanocarriers and a considerable decrease in the diffusion process.

The most important was the amount of TA recovered from mucosa after the application of the different formulations as the purpose of nasal application of TA was to reduce the nasal absorption and increase the contact time within the inflamed mucosa. All the elaborated nanocarriers showed higher mucosal retention of TA compared with commercial Nasacort ${ }^{\circledR}$. NC showed the highest mucosal retention of TA, as $49 \%$ of initial dose was retained in mucosa after 24 hours. NE, NLC, and Nasacort ${ }^{\circledR}$ showed much lower mucosal retention than the NC (Figure 6).

The highest mucosal retention of TA offered by the NC could be attributed to the presence of polymeric Eudragit coat, which permitted the retention of drug within mucosa. The low flux rate obtained from the polymer-based NC indicated their higher tendency to exert a localized effect on the mucosa rather than a permeation-enhancing effect.

\section{Histopathological assessment of local toxicity on nasal mucosa}

All the investigated nanocarriers were subjected to the nasal toxicity study to evaluate their safety. Figure 7 illustrates the histopathological condition of the nasal mucosa after treatment with PBS pH 6.4 (negative control), isopropyl alcohol (positive control), NC, NE, NLC, TA suspension, and Nasacort ${ }^{\circledR}$.

The nasal mucosa treated with PBS showed intact normal mucosal surface lined with stratified squamous epithelium. On the other hand, the mucosa treated with isopropyl alcohol (mucociliary toxic agent) showed complete surface ulceration lined by granulation tissue. Specimens treated by TA suspension or Nasacort ${ }^{\circledR}$ showed mild surface erosion with chronic inflammatory cells formed of lymphocytes and histiocytes. Specimens treated with NLC and NC showed mild inflammatory cells compared to those treated with the drug suspension and Nasacort ${ }^{\circledR}$. However, the NE-treated mucosa showed surface erosion with chronic inflammatory cells. These results revealed the safety of NC and NLC for nasal administration compared with that of the commercially 


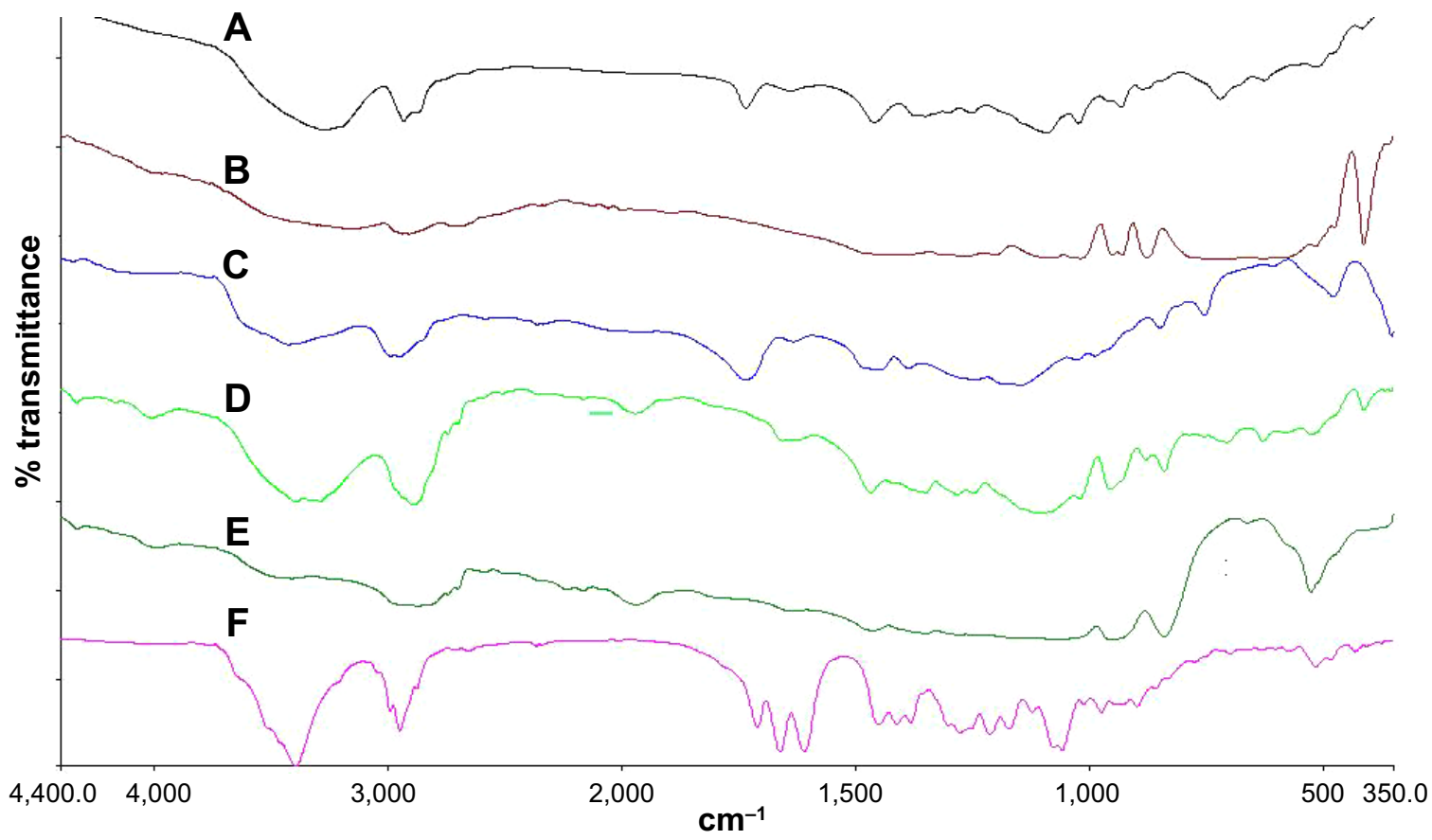

Figure 4 FTIR spectrum of mannitol (A), Eudragit RSI00 (B), Poloxamer I88 (C), TA (D), physical mixture (E), and TA-loaded NC (F).

Abbreviations: FTIR, Fourier-transform infrared spectroscopy; NC, nanocapsule; TA, triamcinolone acetonide.

available nasal spray. This might be due to the encapsulation of the drug, thereby reducing its direct irritant effect on the mucosal tissue. However, the NE is not recommended.

\section{Mucin interaction}

Mucoadhesion studies were performed for the dispersion of the nanocarriers with mucin over a period of 2 hours, by measuring the turbidity at $650 \mathrm{~nm}$ (ABS), change in the particle size, and ZP (Figure 8). The absorbance of the reference mucin dispersion did not significantly deviate from zero (being within the range of 0.001-0.01). However,

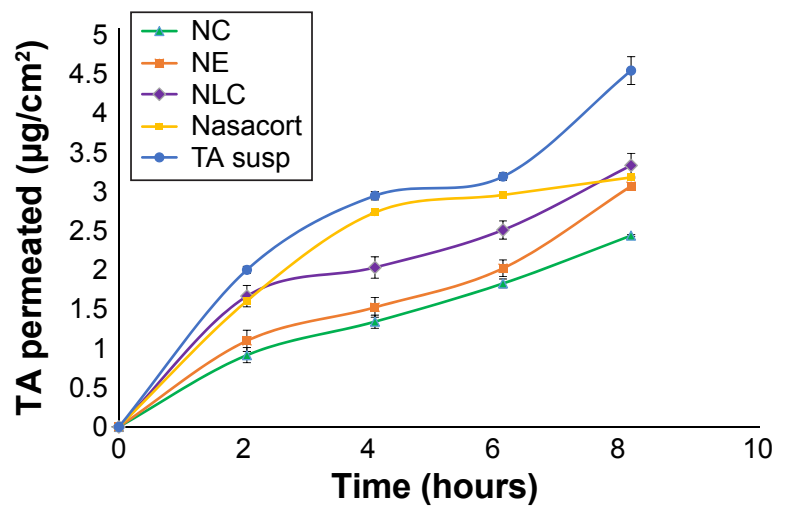

Figure 5 Ex vivo permeation of TA from NC, NE, NLC, Nasacort ${ }^{\circledR}$, and TA susp. Abbreviations: NC, nanocapsule; NE, nanoemulsion; NLC, nanostructured lipid carrier; susp, suspension; TA, triamcinolone acetonide. significantly higher values of absorbance were observed at any time point for the nanocarrier aqueous dispersions. This is a sufficient indication of the nanocarrier motion. In the case of $\mathrm{NC}$ and $\mathrm{NE}$, the absorbance did not significantly increase straight after their addition to the mucin-containing medium $(P<0.05)$ (Figure 8A), while for NLC, the absorbance of nanodispersion/mucin blend increased significantly $(P<0.05)$ with time. This was a good indication of nanoparticle motion that decreased the incidence of NLC and mucin interaction.

Nanoparticle-mucin interactions were also confirmed by the particle size and ZP measurements (Figure $8 \mathrm{~B}$ and $\mathrm{C}$ ). In particular, the strongly positive $\mathrm{ZP}$ of the $\mathrm{NC}$ dispersion was inverted to a slightly negative value by time it was added to the mucin dispersion. This was not the same in the case NLC and NE, as their ZP was not significantly changed in the presence or absence of mucin.

Furthermore, the particle size of NC dispersion increased markedly upon addition to the mucin solution with time, whereas, for NLC and NE, the particle size did not show a significant increase upon time after addition to the mucin solution. ${ }^{17,26}$

The elaborated NC outer shell is constructed of Eudragit RS100, which exhibits positive charge because of its quaternary ammonium groups, whereas mucins are negatively charged at physiological $\mathrm{pH}^{27}$ 


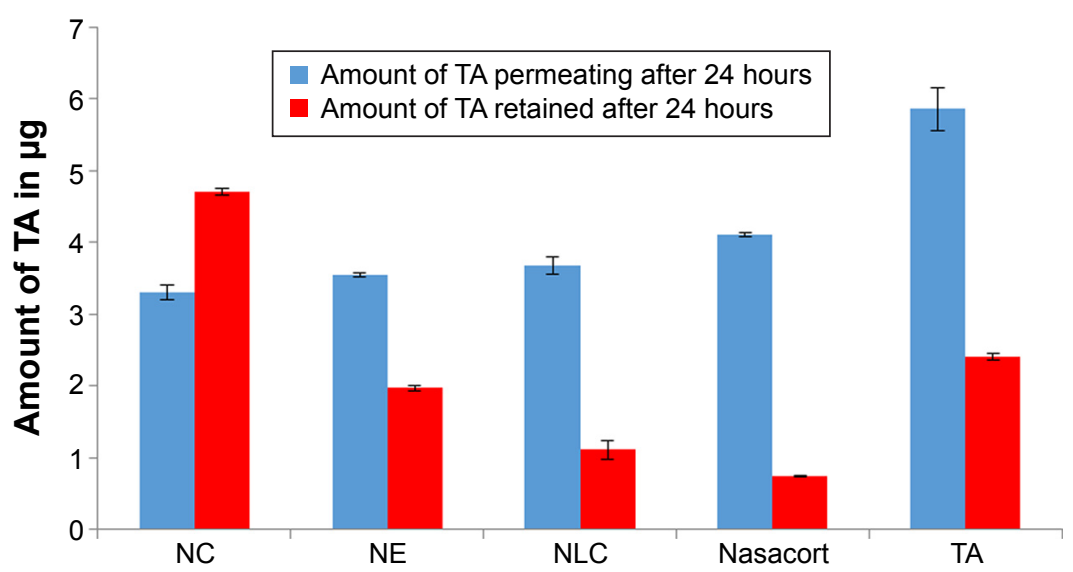

Figure 6 Amount of TA permeating through and retained in the nasal mucosa after 24 hours of application of the different nanocarriers, Nasacort ${ }^{\circledR}$, and TA suspension. Abbreviations: NC, nanocapsule; NE, nanoemulsion; NLC, nanostructured lipid carrier; TA, triamcinolone acetonide.

These charges enhanced a predicted and well-tolerated interaction between NC and nasal mucin. Mucins are highly glycosylated glycoproteins with a large peptide backbone and oligosaccharides as side chains. Their protein backbone is characterized by the presence of repeating sequences rich in serine, threonine, and proline residues. Many of the O-linked oligosaccharide side chains are often terminated in sialic acid, sulfonic acid, or L-fructose.

As a result, the interaction led to decrease in $\mathrm{NC}$ motion, which in turn will decrease their wash out by nasal mucociliary motion. This was obvious in the increase in the particle size and change in the ZP of NC negatively upon interaction with mucin. Eudragit ${ }^{\circledR}$ itself had mucoadhesive properties to other mucosal surfaces, which may give it superiority to adhere to nasal mucosa as well..$^{28,29}$

NLC and NE did not exhibit an obvious interaction with mucin. This was indicated by the movement of the nanocarriers that resulted in an increase in the absorbance of the mucin-nanocarrier blend. In addition, the particle size and ZP of the NLC and NE did not differ from their initial state before addition to the mucin solution. All these findings suggested the absence of interaction between NLC or NE and mucin. In addition, measuring the $\mathrm{ZP}$ of mucin/TA dispersion and the mucin/Nasacort ${ }^{\circledR}$ dispersion revealed the absence of any
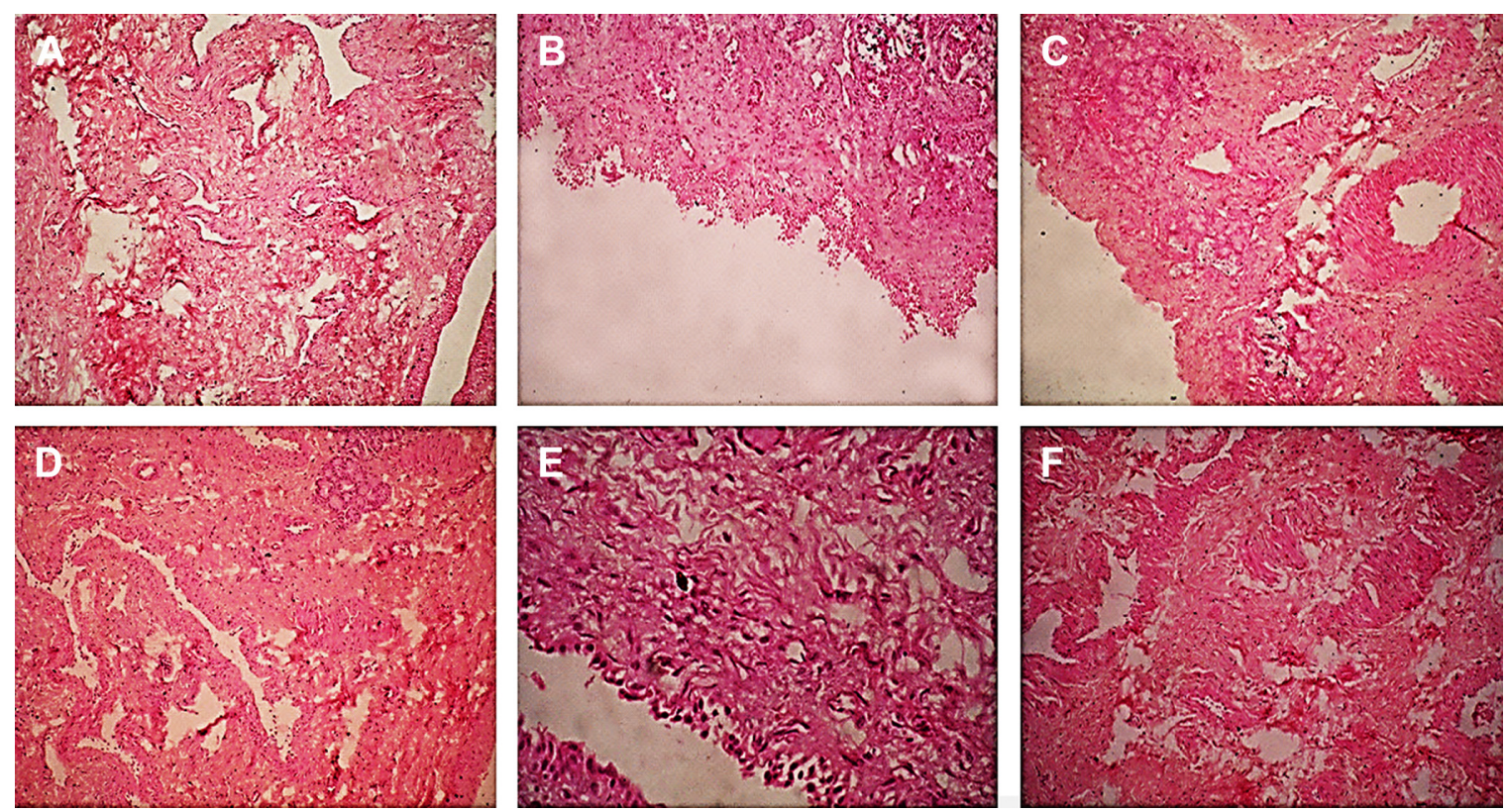

Figure 7 Histopathological condition (magnification 40x) of nasal mucosa after 2 hours exposure to PBS pH 6.4 (negative control) (A), IPA (positive control) (B), Nasacort ${ }^{\circledR}$ (C), NC (D), NE (E), and NLC (F).

Abbreviations: IPA, isopropyl alcohol; NC, nanocapsule; NE, nanoemulsion; NLC, nanostructured lipid carrier; PBS, phosphate-buffered saline. 
A

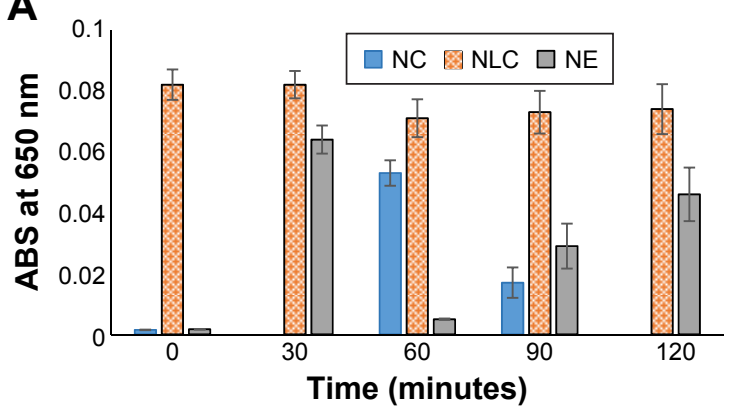

B

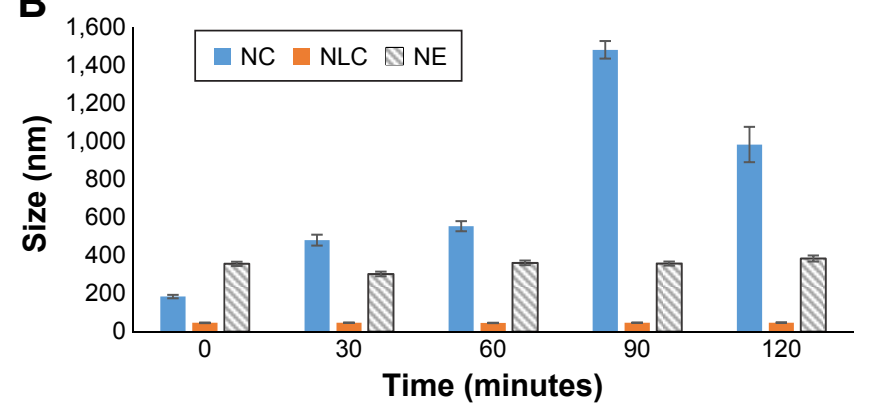

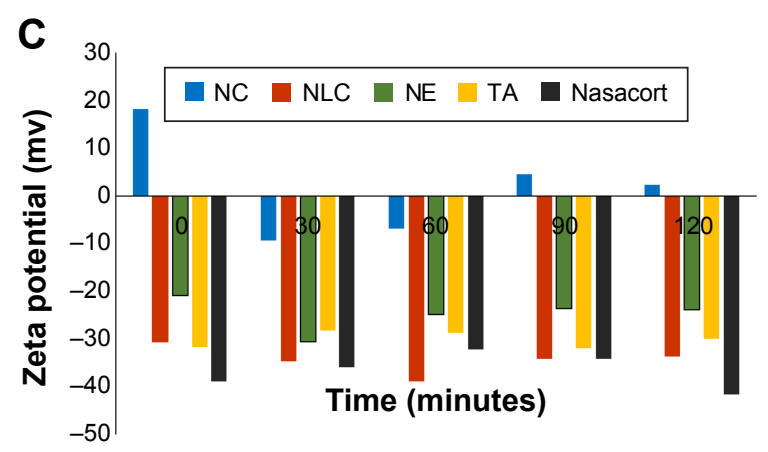

Figure 8 In vitro assessment of nanoparticles interaction with mucin (A) turbidimetry at $650 \mathrm{~nm}$, (B) particle-size analysis of NP in mucin, and (C) zeta potential of NP in mucin.

Abbreviations: ABS, absorbance; NC, nanocapsule; NE, nanoemulsion; NLC, nanostructured lipid carrier; NP, nanoparticle.

interaction between them ( $\mathrm{ZP}$ of mucin solution is $-26.5 \mathrm{mv}$ ). This further proved that the elaborated lipid-core Eudragit ${ }^{\circledR}$ NCs possess superior nasal mucosal adhesion.

Five theories have been suggested to play a major role in bioadhesion, namely, adsorption, diffusion, electronic, fracture, and wetting theories. The mucoadhesion theory proposes that the adhesion of a polymer to a biological tissue results from 1) primary chemical bonds that are somewhat permanent and therefore undesirable in bioadhesion and 2) van der Waals, hydrogen, hydrophobic, and electrostatic forces, which form secondary chemical bonds. ${ }^{30}$ The electronic theory is based on the electron transfer between different electronic properties of the mucoadhesive polymer and the mucus glycoprotein. A charged double layer at the interface of the mucus and the polymer was formed that caused forces of attraction in this region. ${ }^{27}$

\section{In vivo nasal drug deposition}

The amount of TA remaining in the nasal cavity was determined after the application of each of the nanocarriers, TA suspension, and Nasacort ${ }^{\circledR}$ in rat's nasal cavity.

Results revealed that the percentage of drug remaining in the nasal cavity after 10 minutes was $\sim 98 \%$ for NC incorporating $\mathrm{TA}$. On the other hand, the remaining drug percentage was lesser in the case of TA suspension and Nasacort ${ }^{\mathbb{R}}$.
For NE and NLC, the percentage of drug remaining after 10 minutes was $83 \%$ and $89 \%$, respectively. Similarly, after 60 minutes, the TA-loaded NC showed significantly $(P<0.05)$ higher drug residence in the nasal cavity demonstrated by the corresponding lower amount of TA in the nasal wash (Figure 9).

The polymeric coat NC exhibited the highest retention time within the nasal cavity. This could be attributed to Eudragit RS100 poly(ethyl acrylate-co-methyl methacrylateco-trimethylammonioethyl methacrylate chloride), which is a poly methacrylic acid derivative that has high mucoadhesive property. ${ }^{31}$

It has been reported that higher nasal drug absorption is accompanied with a decreased rate of mucociliary clearance of the nose. In other words, increased mucoadhesion leads to increased drug absorption by increasing the contact time with nasal mucosa. ${ }^{30}$

The elaborated $\mathrm{NC}$ had the best mucin interaction parameters, the highest mucosal drug retention, and the highest nasal deposition, which in turn will increase the effectiveness of the nasal TA treatment of rhinitis.

\section{Stability study of TA-loaded nanocarriers}

The physical stability of the drug-loaded nanoparticles is an important parameter to be studied. As for the particle-size 


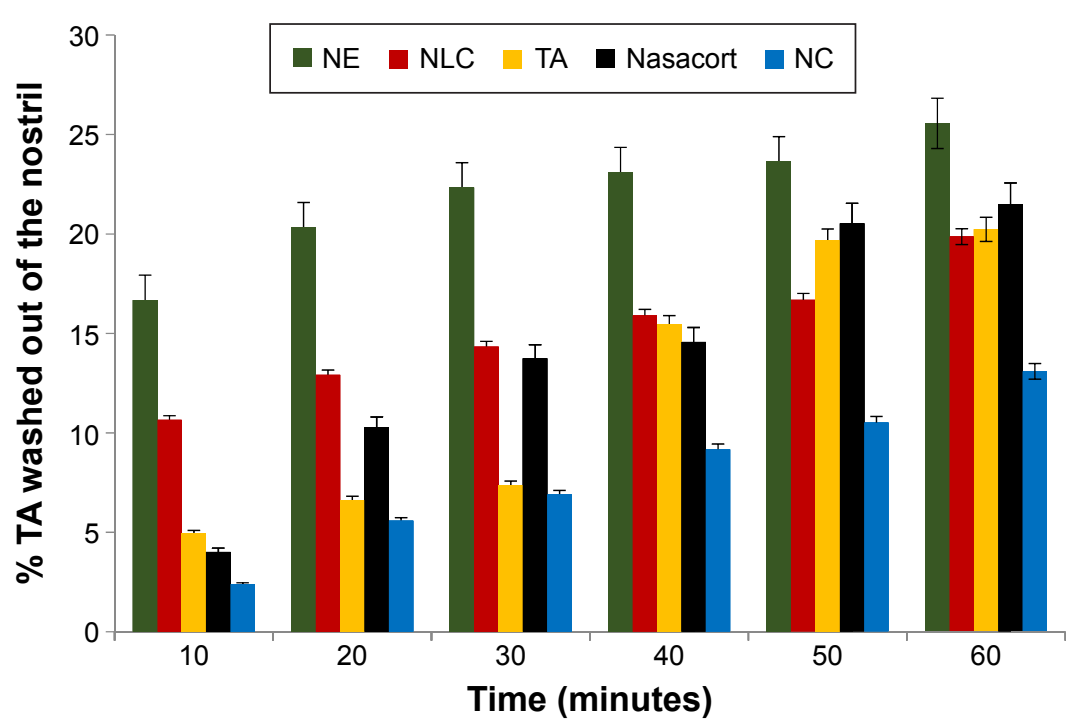

Figure 9 Percentage of TA washed out of the nasal cavity after application of different nanocarriers, TA suspension, and Nasacort ${ }^{\circledR}$. Abbreviations: NC, nanocapsule; NE, nanoemulsion; NLC, nanostructured lipid carrier; TA, triamcinolone acetonide.

measurements, an insignificant fluctuation $(P<0.05)$ in the mean size and PDI was observed for NC (Figure 10). On the other hand, the particle sizes of NE and NLC changed significantly upon storage, which indicated the lack of stability. The ZP values remained above $\pm 20 \mathrm{mV}$ for the three nanocarriers over the 3 -month storage period. The TA content in the nanocarriers did not change significantly upon storage, which gives more evidence on the stability of prepared nanodispersions.

In addition, TA-loaded nanoparticles remained visually unchanged during storage in the referred conditions. No sediment, cream, or free oil was observed in any of the
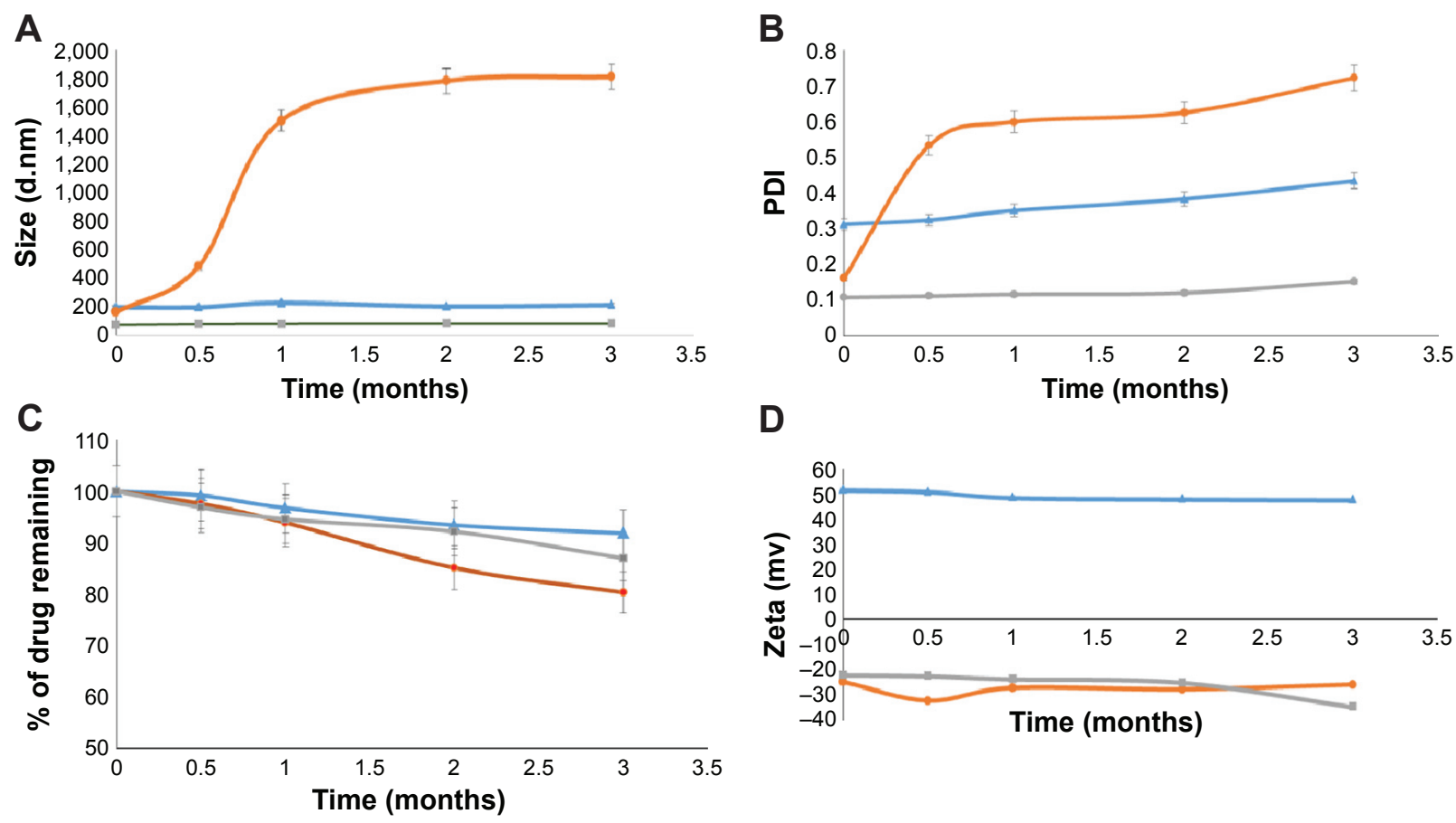

D

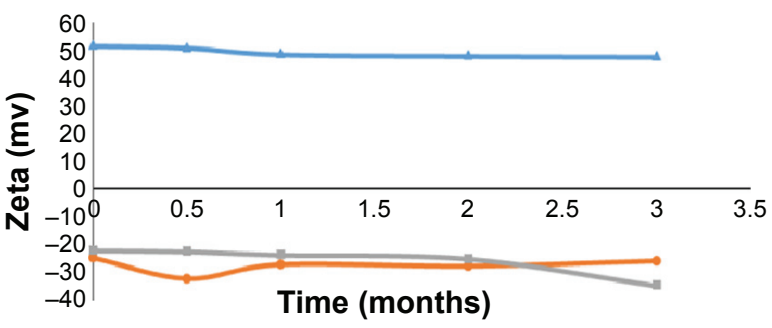

$\because N C \approx N E-N L C$

Figure 10 Stability study parameters: (A) particle size, (B) PDI, (C) percentage of drug remaining, (D) zeta potential of NC, NE, and NLC upon storage for 3 months. Abbreviations: NC, nanocapsule; NE, nanoemulsion; NLC, nanostructured lipid carrier; PDI, polydispersity index. 
formulations. In addition, no TA crystals were observed over the 3-month period.

According to the previously mentioned findings, TAloaded NC formed the most stable nanocarrier of the three prepared formulations giving it superiority for nasal use.

\section{Conclusion}

Polymeric oil-core NC, NE, and NLC have been investigated as local nasal drug delivery systems for TA. Among the investigated systems, the elaborated $\mathrm{NC}$ showed the best mucin interaction parameters, the highest mucosal drug retention, and the highest nasal drug deposition with the least permeation flux. This in turn will increase the effectiveness of nasal TA treatment of rhinitis and reduce the systemic absorption compared with the drug suspension and the marketed product. NC also showed lower mucosal irritation and superior stability compared with NE. TA-loaded NC presented the most promising nanocarrier of all the investigated systems giving it superiority for nasal use compared with lipid-based nanocarriers and commercial product.

Our results suggest that the elaborated polymeric oil-core $\mathrm{NC}$ can be considered an efficient carrier system for localized nasal drug delivery.

\section{Acknowledgments}

The authors thank Gattefossé Company for providing the oils and lipids used in this study and Lipoid Company for providing Lipoid S75, and also Evonik Company for supplying Eudragit RS100.

\section{Disclosure}

The authors report no conflicts of interest in this work.

\section{References}

1. Wallace DV, Dykewicz MS, Bernstein DI, et al. The diagnosis and management of rhinitis: an updated practice parameter. J Allergy Clin Immunol. 2008;122:S1-S84.

2. Blaiss MS, Pediatric allergic rhinitis: physical and mental complications. Allergy Asthma Proc. 2008;29:1-6.

3. Kumar A, Pandey AN, Jain SK. Nasal-nanotechnology: revolution for efficient therapeutics delivery. Drug Deliv. 2014;5:1-13.

4. Singh RM, Kumar A, Pathak K. Mucoadhesive in situ nasal gelling drug delivery systems for modulated drug delivery. Expert Opin Drug Deliv. 2013;10:115-130.

5. Xu X, Shen Y, Wang W, et al. Preparation and in vitro characterization of thermosensitive and mucoadhesive hydrogels for nasal delivery of phenylephrine hydrochloride. Eur J Pharm Biopharm. 2014;88: 998-1004.

6. Berger WE, Meltzer EO. Intranasal spray medications for maintenance therapy of allergic rhinitis. Am J Rhinol Allergy. 2015;29:273-282.

7. Keleş N. Treatment of allergic rhinitis during pregnancy. Am J Rhinol. 2004;18:23-28.

8. Sabzevari A, Adibkia K, Hashemi H, et al. Polymeric triamcinolone acetonide nanoparticles as a new alternative in the treatment of uveitis: in vitro and in vivo studies. Eur J Pharm Biopharm. 2013;84: 63-71.
9. Noronha CM, Granada AF, de Carvalho SM, et al. Optimization of $\alpha$-tocopherol loaded nanocapsules by the nanoprecipitation method. Ind Crops Prod. 2013;50:896-903.

10. Mendes AI, Silva AC, Catita JA, Cerqueira F, Gabriel C, Lopes CM. Miconazole-loaded nanostructured lipid carriers (NLC) for local delivery to the oral mucosa: improving antifungal activity. Colloids Surf $B$ Biointerfaces. 2013;111:755-763.

11. Berger N, Sachse A, Bender J, Schubert R, Brandl M. Filter extrusion of liposomes using different devices: comparison of liposome size, encapsulation efficiency, and process characteristics. Int J Pharm. 2001; 223:55-68.

12. Kadam RS, Tyagi P, Edelhauser HF, Kompella UB. Influence of choroidal neovascularization and biodegradable polymeric particle size on transscleral sustained delivery of triamcinolone acetonide. Int J Pharm. 2012;434:140-147.

13. Peter Christoper GV, Vijaya Raghavan C, Siddharth K, Siva Selva Kumar M, Hari Prasad R. Formulation and optimization of coated PLGA - Zidovudine nanoparticles using factorial design and in vitro in vivo evaluations to determine brain targeting efficiency. Saudi Pharm J. 2014;22:133-140.

14. Schmidt MC, Simmen D, Hilbe M, et al. Validation of excised bovine nasal mucosa as in vitro model to study drug transport and metabolic pathways in nasal epithelium. J Pharm Sci. 2000;89:396-407.

15. Pund S, Rasve G, Borade G. Ex vivo permeation characteristics of venlafaxine through sheep nasal mucosa. Eur J Pharm Sci. 2013;48: 195-201.

16. Lalani J, Baradia D, Lalani R, Misra A. Brain targeted intranasal delivery of tramadol: comparative study of microemulsion and nanoemulsion. Pharm Dev Technol. 2014;17:1-10.

17. Ungaro F, d'Angelo I, Coletta C, et al. Dry powders based on PLGA nanoparticles for pulmonary delivery of antibiotics: modulation of encapsulation efficiency, release rate and lung deposition pattern by hydrophilic polymers. J Control Release. 2012;157:149-159.

18. Rossi S, Ferrari F, Bonferoni MC, Caramella C. Characterization of chitosan hydrochloride-mucin interaction by means of viscosimetric and turbidimetric measurements. Eur J Pharm Sci. 2000;10:251-257.

19. Faraj JA, Hussain AA, Aramaki Y, Iseki K, Kagoshima M, Dittert LW. Mechanism of nasal absorption of drugs. III: nasal absorption of leucine enkephalin. J Pharm Sci. 1990;79:698-702.

20. Aikawa K, Mitsutake N, Uda H, et al. Drug release from $\mathrm{pH}$-response polyvinylacetal diethylaminoacetate hydrogel, and application to nasal delivery. Int J Pharm. 1998;168:181-188.

21. Wilson AM, Dempsey OJ, Sims EJ, Lipworth BJ. A comparison of topical budesonide and oral montelukast in seasonal allergic rhinitis and asthma. Clin Exp Allergy. 2001;31:616-624.

22. Puglia C, Damiani E, Offerta A, et al. Evaluation of nanostructured lipid carriers (NLC) and nanoemulsions as carriers for UV-filters: characterization, in vitro penetration and photostability studies. Eur $J$ Pharm Sci. 2014;51:211-217.

23. Santos SS, Lorenzoni A, Pegoraro NS, et al. Formulation and in vitro evaluation of coconut oil-core cationic nanocapsules intended for vaginal delivery of clotrimazole. Colloids Surf B Biointerfaces. 2014; 116:270-276.

24. Gonzalez-Mira E, Egea MA, Garcia ML, Souto EB. Design and ocular tolerance of flurbiprofen loaded ultrasound-engineered NLC. Colloids Surf B Biointerfaces. 2010;81:412-421.

25. Araújo J, Gonzalez-Mira E, Egea MA, Garcia ML, Souto EB. Optimization and physicochemical characterization of a triamcinolone acetonide-loaded NLC for ocular antiangiogenic applications. Int $J$ Pharm. 2010;393:167-175.

26. Takeuchi H, Thongborisute J, Matsui Y, Sugihara H, Yamamoto H, Kawashima Y. Novel mucoadhesion tests for polymers and polymercoated particles to design optimal mucoadhesive drug delivery systems. Adv Drug Deliv Rev. 2005;57:1583-1594.

27. Salamat-Miller N, Chittchang M, Johnston TP. The use of mucoadhesive polymers in buccal drug delivery. Adv Drug Deliv Rev. 2005;57: 1666-1691. 
28. Karn PR, Vanić Z, Pepic I, Skalko-Basnet N. Mucoadhesive liposomal delivery systems: the choice of coating material. Drug Dev Ind Pharm. 2011;37:482-488

29. Frank LA, Sandri G, D’Autilia F, et al. Chitosan gel containing polymeric nanocapsules: a new formulation for vaginal drug delivery. Int J Nanomed. 2014;9:3151-3161.
30. Ugwoke M, Agu R, Verbeke N, Kinget R. Nasal mucoadhesive drug delivery: background, applications, trends and future perspectives. Adv Drug Deliv Rev. 2005;57:1640-1665.

31. Chun M-K, Cho C-S, Choi H-K. A novel mucoadhesive polymer prepared by template polymerization of acrylic acid in the presence of poloxamer. J Appl Polym Sci. 2001;79:1525-1530.
International Journal of Nanomedicine

\section{Publish your work in this journal}

The International Journal of Nanomedicine is an international, peerreviewed journal focusing on the application of nanotechnology in diagnostics, therapeutics, and drug delivery systems throughout the biomedical field. This journal is indexed on PubMed Central, MedLine, CAS, SciSearch $®$, Current Contents $® /$ Clinical Medicine,

\section{Dovepress}

Journal Citation Reports/Science Edition, EMBase, Scopus and the Elsevier Bibliographic databases. The manuscript management system is completely online and includes a very quick and fair peer-review system, which is all easy to use. Visit http://www.dovepress.com/ testimonials.php to read real quotes from published authors.

Submit your manuscript here: http://www.dovepress.com/international-journal-of-nanomedicine-journal 